\title{
RESISTÊNCIA E DUCTILIDADE DE COLUNAS CURTAS DE CONCRETO ARMADO REFORÇADAS COM COMPÓSITO DE RESINA E FIBRAS DE CARBONO
}

\author{
SARAIVA, REBECA M. DIAS DE CARVALHO \\ Professora D.Sc. \\ Instituto Militar de Engenharia \\ Rio de Janeiro; Brasil \\ rebecadias@gmail.com
}

TEIXEIRA, ANA MARIA ABREU

Professora D.Sc.

Instituto Militar de Engenharia

Rio de Janeiro; Brasil

anam@ime.eb.br

\author{
CARNEIRO, LUIZ ANTONIO VIEIRA \\ Professor Adjunto \\ Universidade Federal Fluminense \\ Rio de Janeiro; Brasil \\ luizcarneiro@id.uff.br
}

\section{RESUMO}

Este trabalho tem por objetivo apresentar resultados de um estudo experimental sobre a resistência e ductilidade de colunas curtas de concreto armado reforçadas com compósito de resina e fibras de carbono (CFRP) colado externamente. Todas as colunas de concreto armado de $150 \mathrm{~mm}$ x $300 \mathrm{~mm}$ de dimensões foram submetidas ao ensaio de compressão simples, cujos parâmetros variados foram o número de camadas de reforço de CFRP $(0,1$ e 2$)$ e o espaçamento entre estribos (30 $\mathrm{mm}$ ou $60 \mathrm{~mm}$ ). Constatou-se que o reforço com folhas unidirecionais de compósito de resina e fibras de carbono aumenta a resistência do núcleo de concreto e a deformação última das colunas curtas de concreto armado em até cerca de $236 \%$ e $993 \%$. Também verificou-se que quanto menor o espaçamento entre os estribos, para uma mesma taxa de reforço, maiores os valores de resistência e de deformação última das colunas curtas de concreto armado.

Palavras-chave: reforço, colunas curtas, concreto armado, fibra de carbono.

\section{ABSTRACT}

This paper aims to present results of an experimental study on the strength and ductility of reinforced concrete short columns strengthened with carbon fiber reinforced plastic (CFRP) externally glued. All of reinforced concrete columns of $150 \mathrm{~mm}$ x $300 \mathrm{~mm}$ in dimensions were submitted to axial compression test, in which number of CFRP layer ( 0 , 1 and 2) and stirrups spacing $(30 \mathrm{~mm}$ or $60 \mathrm{~mm}$ ) were the varied parameters. It was found that the strengthening with carbon fiber reinforced plastic sheet increases the concrete core strength and the ultimate strain of reinforced concrete short columns up to about $236 \%$ and $993 \%$. It was also verified that smaller stirrups spacing, for the same reinforcement rate, higher strength and ultimate strains values of reinforced concrete short columns.

Keywords: strengthening, short columns, reinforced concrete, carbon fiber.

\section{INTRODUÇÃO}

O concreto armado é o material mais largamente usado na construção civil, devido à facilidade com que elementos estruturais podem ser executados, em variedade de formas e tamanhos.

Contudo, ao longo do tempo, o concreto armado pode sofrer deteriorações ou mudanças de destinação. Erros de projeto ou de execução ou aumento da solicitação da estrutura podem vir a comprometer a capacidade resistente dos elementos estruturais.

Para que o elementro estrutural de concreto armado continue atendendo às necessidades dos usuários, mesmo após as mudanças sofridas, o reforço estrutural é uma das técnicas que pode ser empregada. 
Exemplos comuns de necessidade de reforço de estruturas de concreto armado são as obras de arte especiais, tais como pontes e viadutos. Até o ano de 1985, as pontes foram projetadas com um trem-tipo de $360 \mathrm{kN}$ e, atualmente, o tremtipo é de $450 \mathrm{kN}$.

Várias são as técnicas de reforço de elementos estruturais de concreto, cuja escolha depende das causas e extensão dos danos, da disponibilidade dos materiais de reforço, equipamentos e mão-de-obra especializada, do estudo de viabilidade técnico-econômica, da utilização ou não da estrutura durante a obra e da classe de agressividade do ambiental.

Com o avanço da ciência dos materiais, impulsionado pela demanda de materiais mais leves, duráveis e resistentes, surgiram os materiais compósitos de resina e fibras, de diferentes tipos, que começaram a ser usados nas indústrias aeronáutica, aeroespacial, militar, naval, automobilística e de materiais esportivos.

Atualmente, a construção civil utiliza os materiais compósitos na forma de folhas ou tecidos em seus serviços de reforço, por serem materiais versáteis e de fácil aplicação, além de possuírem elevadas resistências à corrosão e à fadiga, bem como bom desempenho no que diz respeito à fluência e à relaxação.

Com base nos estudos de HOSOTANI et al. (1997), MATTHYS (2000), COLE e BELARBI (2001), PESSIKI et al. (2001), WANG e RESTREPO (2001), PAULA e SILVA (2002), ILKI et al. (2008), CHASTRE e SILVA (2010), e MAADDAWY et al. (2010), foi possível verificar que o desempenho do confinamento do núcleo do concreto do pilar é influenciado pela qualidade do compósito e do envelopamento e cresce com o aumento do número de camadas de reforço, em contrapartida diminui com o incremento das dimensões da seção transversal.

Os ganhos de resistência foram maiores para pilares curtos de concreto armado, feitos com concreto de menor valor de resistência à compressão (DEMERS e NEALE, 1999; ILKI et al., 2008; BENZAID et al., 2010; CHASTRE e SILVA, 2010) e de seção transversal circular, enquanto os maiores ganhos de ductilidade, para pilares esbeltos de concreto armado e de seção transversal quadrada e retangular.

O arredondamento das arestas nos pilares de seção quadrada e retangular deve ser realizado e aumentou significativamente a resistência dos mesmos, mas não teve influência sobre a ductilidade, segundo resultados experimentais desenvolvidos por MATTHYS (2000), COLE e BELARBI (2001), PAULA e SILVA (2002), ILKI et al. (2008) e SILVA (2011).

Na maioria dos casos, a curva tensão-deformação do concreto confinado com compósito de resina e fibra de carbono é aproximadamente uma curva bilinear crescente com zona de transição, na qual o ramo elástico não sofre alteração substancial em relação à curva do concreto não confinado, enquanto o ramo plástico é função do nível de confinamento (CARNEIRO, 2004).

Apresentam-se neste trabalho resultados experimentais sobre a resistência e ductilidade de colunas curtas de concreto armado reforçadas com compósito de resina e fibra de carbono (CFRP) colado externamente. Todas as colunas de concreto armado de $150 \mathrm{~mm}$ x $300 \mathrm{~mm}$ de dimensões foram submetidas ao ensaio de compressão simples, cujos parâmetros variados foram o número de camadas de reforço de CFRP $(0,1$ e 2$)$ e o espaçamento entre estribos (30 mm ou $60 \mathrm{~mm})$.

\section{PROGRAMA EXPERIMENTAL}

Objetivou-se investigar o comportamento de colunas curtas de concreto armado confinadas com folhas de compósito de resina e fibras de carbono em termos de resistência à compressão e de deformação.

Foram ensaiados 18 espécimes de concreto armado, todos com diâmetro de $150 \mathrm{~mm}$ e altura de $300 \mathrm{~mm}$, sendo 6 com uma camada de reforço, outros 6 com duas de camadas de reforço e os 6 restantes não reforçados (de referência). Metade dos 18 espécimes tinha espaçamento entre estribos igual a $30 \mathrm{~mm}$, enquanto a outra metade, $60 \mathrm{~mm}$.

Todos os espécimes foram submetidos à compressão centrada, com taxa de carregamento constante de $0,22 \mathrm{MPa} / \mathrm{s}$ até a ruptura, tendo sido monitorados os valores de carga e de deformação nas direções longitudinal e transversal. 


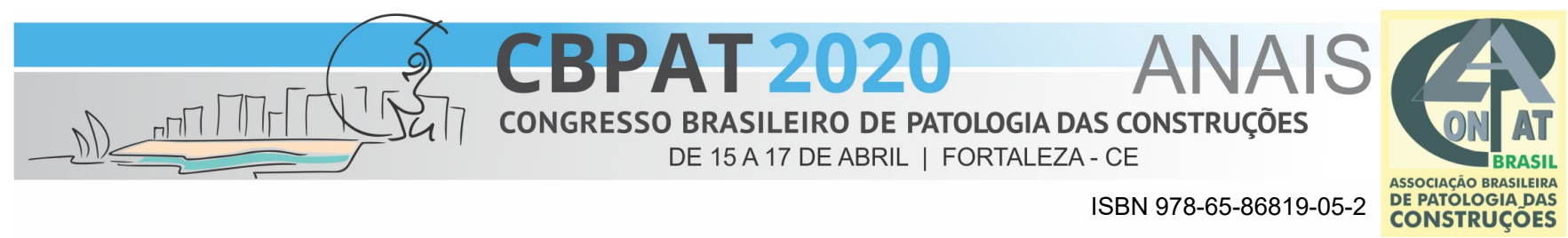

\subsection{Materiais}

\subsubsection{Concreto}

A fim de se obter resistência média do concreto à compressão igual a aproximadamente $30 \mathrm{MPa}$, a composição do concreto utilizado, em massa, foi 1: 2,18: 2,87 (cimento: areia: agregado graúdo), sendo o consumo de cimento de $352 \mathrm{~kg} / \mathrm{m}^{3}$ de concreto. A relação água-aglomerante e o abatimento do tronco de cone do concreto foram de 0,54 e 35 $\mathrm{mm}$.

Utilizaram-se cimento CPII-E-32, areia natural de rio lavada e agregado graúdo britado do tipo gnaisse com dimensões máximas características de 9,5 mm (50\% em massa de brita 0) e $19 \mathrm{~mm}$ (50\% em massa de brita 1$)$.

Após 24 horas, os espécimes de concreto foram desformados e imersos em tanque com água saturada de cal por sete dias, sendo então mantidos sob as condições ambientes do Laboratório de Materiais de Construção e Concreto do Instituto Miliar de Engenharia (IME). Foram executados os ensaios de resistência à compressão do concreto conforme a norma NBR 5739 (ABNT, 2018).

\subsubsection{Armadura interna}

Em todos os espécimes foi utilizada armadura longitudinal com 4 barras de aço tipo CA-50 de 10 mm de diâmetro, o que equivale a uma taxa geométrica de $1,78 \%$.

Os espécimes, que continham armadura transversal com barras de aço tipo CA-60 de $5 \mathrm{~mm}$ de diâmetro espaçadas a cada $60 \mathrm{~mm}$, apresentaram taxa de armadura volumétrica de 1,01\%, enquanto os espécimes com barras de mesmo diâmetro espaçadas a cada $30 \mathrm{~mm}$, taxa de armadura volumétrica de 2,02\%.

A Figura 1 mostra o detalhe das armaduras internas dos espécimes ensaiados e a Figura 2 ilustra as armaduras formadas por barras de aço utilizadas nos espécimes.

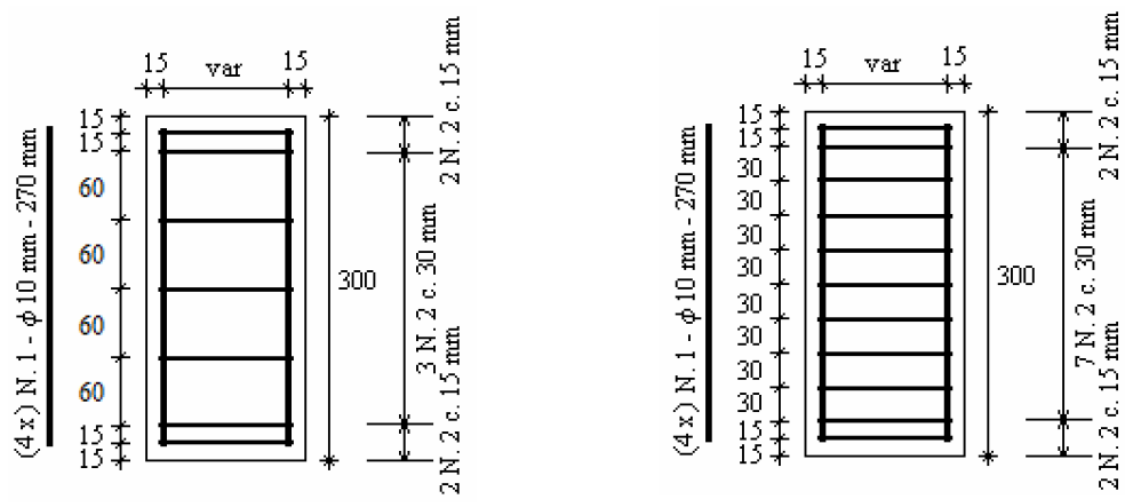

Perfil longitudinal (cotas em $\mathrm{mm})$
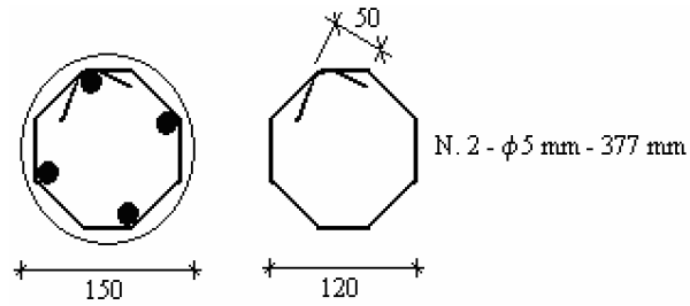

Seção transversal (cotas em mm)

Figura 1: Detalhe das armaduras internas dos espécimes ensaiados 


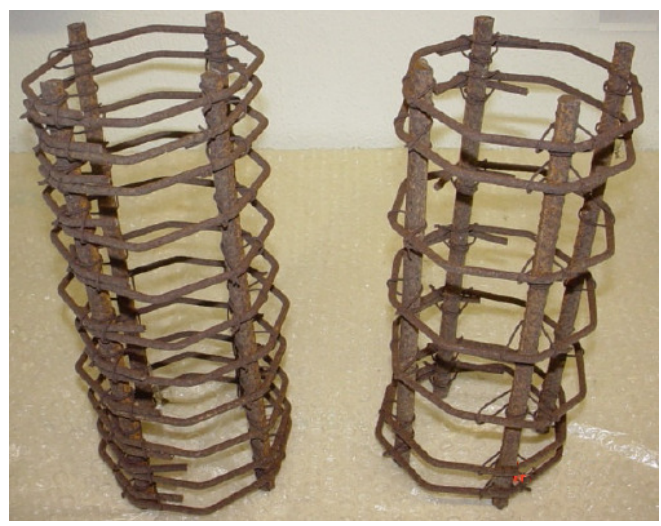

Figura 2: Armaduras longitudinal e transversal de barras de aço

\subsubsection{Resina de imprimação}

Antes da colagem das folhas de compósito de fibras de carbono, os espécimes de concreto armado foram previamente limpos com álcool. Em seguida foi aplicada a resina de imprimação Tec-Poxi PR da RheoSet, cuja função era garantir a perfeita aderência da camada de resina epóxi ao substrato de concreto (DIOGO, 2010). A Tabela 1 apresenta as principais características da resina utilizada.

Tabela 1 - Especificações da resina Tec-Poxi PR da RheoSet

\begin{tabular}{c|c}
\hline Característica & Valor \\
\hline Aspecto & Cor incolor \\
\hline Viscosidade & $65 \mathrm{~s} \mathrm{a} 75 \mathrm{~s}(\mathrm{CF} 4)$ \\
\hline Massa específica & $1,050 \mathrm{~g} / \mathrm{cm}^{3}$ \\
\hline Sólido por volume & Mínimo de $98 \%$ \\
\hline Pot life (tempo de vida útil da mistura) & Mínimo de $40 \mathrm{~min}$ \\
\hline Secagem ao toque & 4 horas máximo \\
\hline Secagem ao manuseio & 6 horas máximo \\
\hline Secagem completa & 10 horas máximo \\
\hline Cura inicial & 7 dias \\
\hline Alongamento por ruptura & $0,300 \mathrm{~mm} / \mathrm{mm}$ \\
\hline Resistência à tração & $20 \pm 2 \mathrm{MPa}$ após 24 horas \\
\hline Rugosidade do substrato & $60 \mathrm{a} 80 \mathrm{~m}$ mírons \\
\hline Rendimento teórico & $250 \mathrm{~g} / \mathrm{m}^{2}$ a $350 \mathrm{~g} / \mathrm{m}^{2}$ \\
\hline
\end{tabular}

A resina Tec-Poxi PR é uma resina bi-componente. Antes de utilizá-la, foi necessário misturar os compostos A e B (v. Figura 3) na proporção de 66,7\% e 33,3\% respectivamente, manualmente por 5 minutos.

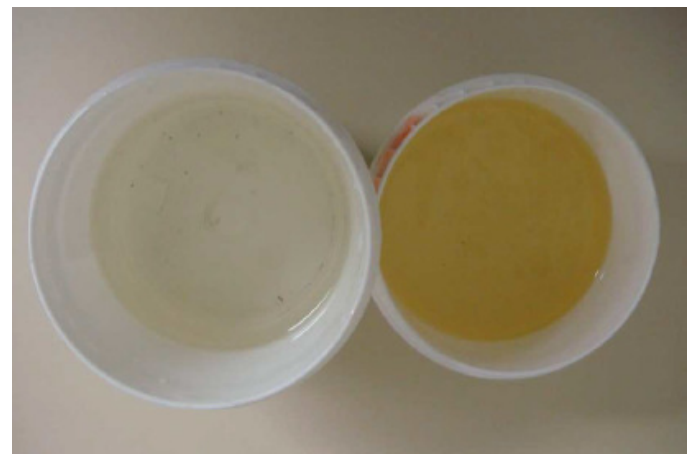

Figura 3: Compostos A e B da resina de imprimação 


\subsubsection{Resina epóxi}

Para a colagem das folhas de compósito de fibras de carbono nos espécimes, foi empregada a resina saturante epóxi Tec-poxi da RheoSet, com as características fornecidas pelo fabricante listadas na Tabela 2.

Tabela 2 - Especificações da resina Tec-Poxi da RheoSet

\begin{tabular}{c|c}
\hline Característica & Valor \\
\hline Aspecto & Cor azul transparente \\
\hline Viscosidade & $70 \mathrm{~s} \mathrm{a} 80 \mathrm{~s}(\mathrm{CF} 4)$ \\
\hline Massa específica & $1,055 \mathrm{~g} / \mathrm{cm}^{3}$ \\
\hline Pot life (tempo de vida útil da mistura) & Mínimo de $40 \mathrm{~min}$ \\
\hline Secagem ao toque & 4 horas máximo \\
\hline Secagem ao manuseio & 6 horas máximo \\
\hline Secagem completa & 10 horas máximo \\
\hline Tempo de cura total & 7 dias \\
\hline Resistência à aderência & $1,5 \mathrm{MPa}$ \\
\hline Alongamento por ruptura & $0,300 \mathrm{~mm} / \mathrm{mm}$ \\
\hline Resistência à tração & $55 \pm 3 \mathrm{MPa}$ após 24 horas \\
\hline Resistência à compressão & Mínimo $60 \mathrm{MPa}$ \\
\hline
\end{tabular}

A resina Tec-Poxi é uma resina bi-componente, pré-dosada, sendo necessário misturar manualmente por 5 minutos os compostos A e B (v. Figura 4), na proporção de $67 \%$ e $33 \%$ respectivamente, conforme recomendação do fabricante, para que o produto pudesse ser utilizado.

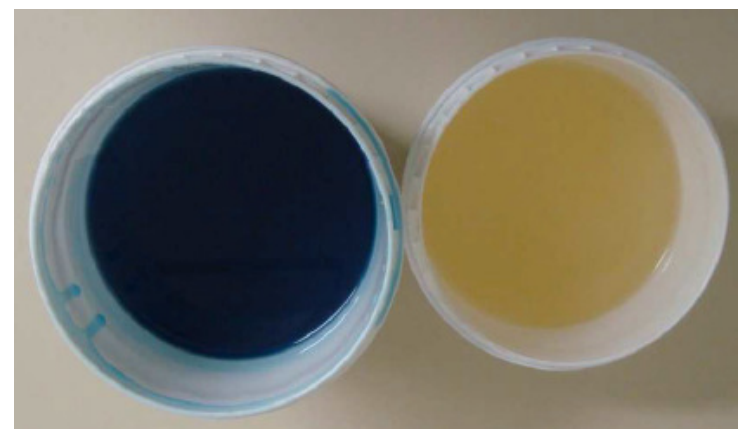

Figura 4: Compostos A e B da resina de imprimação

\subsubsection{Fibras de carbono}

Os espécimes estudados foram confinados com folhas unidirecionais de fibras de carbono Tec-fiber da Rheoset (v. Figura 5), cujas especificações estão reunidas na Tabela 3.

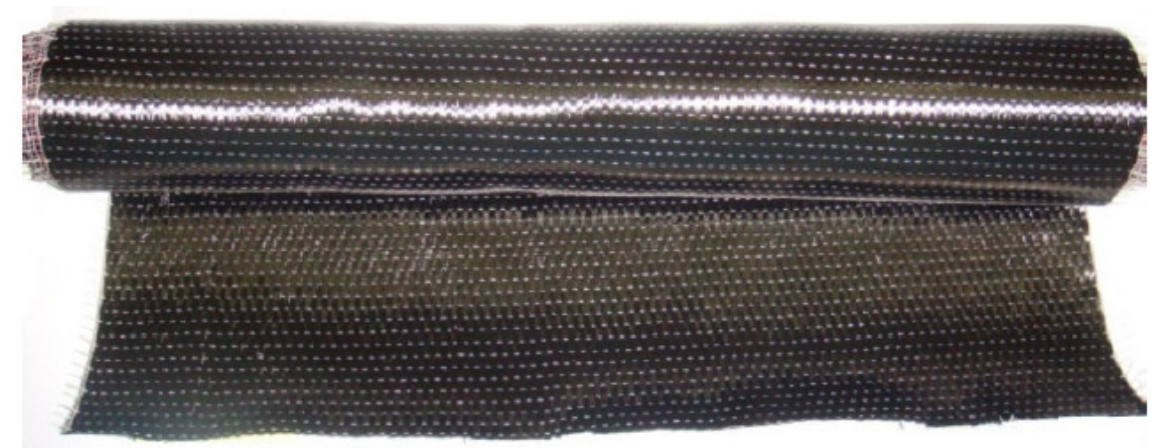

Figura 5: Folha unidirecional de fibras de carbono 
Tabela 3 - Especificações da folha unidirecional de fibras de carbono

\begin{tabular}{c|c}
\hline Característica & Valor \\
\hline Resistência à tração & $3550 \mathrm{MPa}$ \\
\hline Módulo de elasticidade & $235 \mathrm{GPa}$ \\
\hline Deformação de ruptura & $15,5 \%$ \\
\hline Largura da folha & $500 \mathrm{~mm}$ \\
\hline Espessura de projeto da folha & $0,165 \mathrm{~mm}$ \\
\hline Gramatura da folha & $300 \mathrm{~g} / \mathrm{m}^{2}$ \\
\hline
\end{tabular}

\subsubsection{Compósito de resina e fibras de carbono}

O compósito de resina e fibras de carbono foi elaborado com o uso da resina epóxi e da folha unidirecional de fibras de carbono. Primeiramente, a resina epóxi foi aplicada sobra a folha unidirecional de fibras de carbono com o objetivo de saturá-la. Em seguida, com o uso de um rolo de aço, a folha unidirecional de fibras de carbono foi prensada contra uma placa de madeira, após o que ambos os seus lados receberam resina epóxi por meio de um pincel.

Utilizando os mesmos materiais descritos nos itens 2.1.4 e 2.1.5, DIOGO (2010) realizou ensaio de tração uniaxial em espécimes com uma camada de compósito de resina e fibras de carbono de $15 \mathrm{~mm}$ de largura e $250 \mathrm{~mm}$ de comprimento, de acordo com a norma D 3039 M (ASTM, 2000). O detalhe dos espécimes pode ser visto na Figura 6.

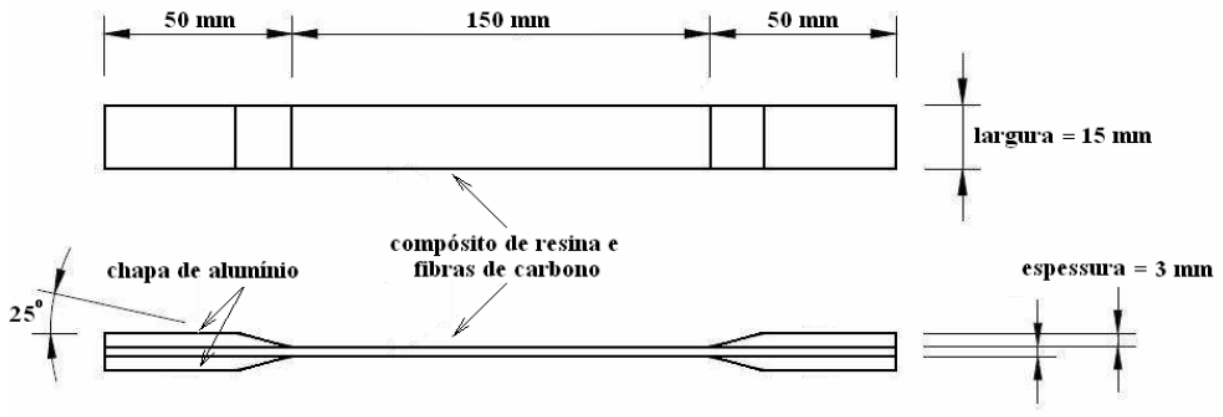

Figura 6: Detalhe do espécime do compósito de resina e fibras de carbono

O ensaio foi realizado na prensa universal EMIC DL 10000 de $100 \mathrm{kN}$ de capacidade do Laboratório de Ensaios Mecânicos do IME (v. Figura 7), cujos resultados estão agrupados na Tabela 4.

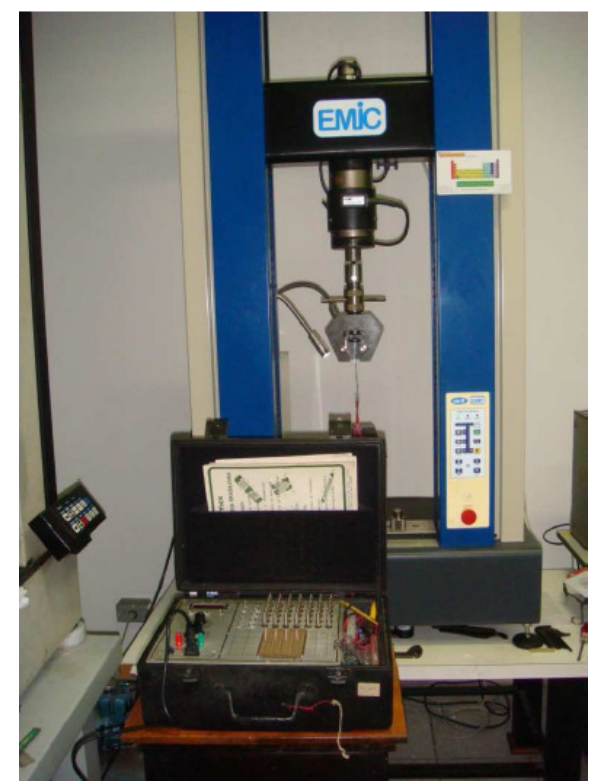

Figura 7: Detalhe da prensa EMIC DL 10000 
Tabela 4 - Resultados do ensaio de tração uniaxial do compósito

\begin{tabular}{c|c|c|c|c}
\hline Espécime & $\begin{array}{c}\text { Carga de ruptura } \\
(\mathrm{N})\end{array}$ & $\begin{array}{c}\text { Resistência à tração } \\
(\mathrm{MPa})\end{array}$ & $\begin{array}{c}\text { Deformação última } \\
(\%)\end{array}$ & $\begin{array}{c}\text { Módulo de elasticidade } \\
(\mathrm{GPa})\end{array}$ \\
\hline 1 & 7248,1 & 2928,5 & 11,9 & 246,1 \\
\hline 2 & 6329,0 & 2557,2 & 9,8 & 260,9 \\
\hline 3 & 8116,6 & 3279,4 & 12,6 & 260,3 \\
\hline Média & 7231,2 & 2921,7 & 11,4 & 255,8 \\
\hline
\end{tabular}

\subsection{Execução do espécimes}

\subsubsection{Formas}

Os pilares curtos de seção transversal circular foram concretados em fôrmas metálicas com dimensões de $150 \mathrm{~mm}$ x $300 \mathrm{~mm}$.

\subsubsection{Concretagem}

O preparo do concreto foi realizado no Laboratório de Materiais de Construção e Concreto do IME, com o uso de uma betoneira de 320 litros. O tempo para homogeneização dos materiais foi de cerca de 5 minutos.

Para o adensamento do concreto, foram utilizados vibradores de imersão com diâmetro de $25 \mathrm{~mm}$, durante e imediatamente após o seu lançamento manual.

Foram colocados sacos plásticos sobre a superfície dos espécimes de concreto fresco para se evitar a evaporação da água a fim de favorecer a cura do concreto.

As fôrmas dos pilaretes de concreto foram retiradas 24 horas após a concretagem. Os espécimes permaneceram em água saturada de cal e, após 7 dias, em condições ambientes do laboratório até a data do ensaio.

\subsubsection{Execução do reforço}

O reforço foi aplicado nos espécimes de concreto armado em etapas: 1) aplicação da resina de imprimação com o uso de um pincel no substrato de concreto; 2) aplicação da primeira camada de resina epóxi com o uso de um pincel; 3) aplicação da folha unidirecional de fibras de carbono pré-impregnada com a resina epóxi por meio de rolo; 4) aplicação da segunda camada de resina epóxi com o uso de um pincel. Para o caso dos espécimes com 2 camadas de reforço, depois da etapa 4 , repetiram-se as etapas 2,3 e 4.

Em todos os espécimes confinados, foi garantida a superposição de cada camada do compósito em um comprimento de $100 \mathrm{~mm}$, sendo o traspasse da camada seguinte defasado de $180^{\circ} \mathrm{em}$ relação ao da camada anterior, como mostra a Figura 8 .

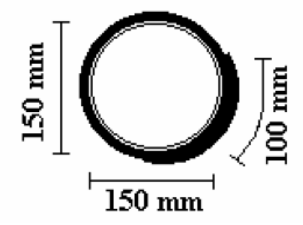

Figura 8: Detalhe do traspasse da camada de reforço nos espécimes

\subsubsection{Instrumentação}

As deformações longitudinal e transversal dos espécimes sem reforço foram medidas com o auxílio de extensômetros elétricos de resistência com base de medição de $80 \mathrm{~mm}$, ao passo que as dos espécimes reforçados foram medidas com extensômetros elétricos de resistência com base de medição de $5 \mathrm{~mm}$. 
Os extensômetros foram colados na superfície externa dos espécimes à meia altura e em posições diametralmente opostas, conforme pode-se notar na Figura 9.

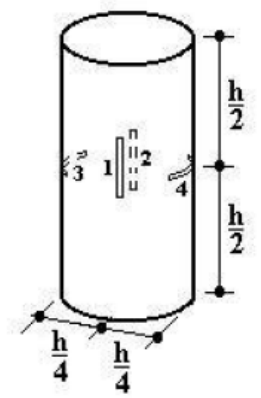

Figura 9: Instrumentação externa dos espécimes ensaiados

\subsubsection{Execução dos ensaios}

Todos os espécimes de concreto armado foram submetidos à tensão normal de compressão uniaxial na direção longitudinal uniforme até a ruptura com uma taxa constante de 0,22 MPa/s, por meio de uma prensa AMSLER com capacidade de 5000 kN do Laboratório de Materiais de Construção e Concreto do IME (v. Figura 10).

O sistema de aquisição de dados modelo P3, da Vishay, foi utilizado para a leitura das deformações medidas pelos extensômetros durante toda a fase de carregamento.

Ambas as superfícies de extremidade, base e topo, dos espécimes foram faceadas, com o objetivo de garantir o paralelismo entre as mesmas e de distribuir uniformemente o carregamento aplicado.

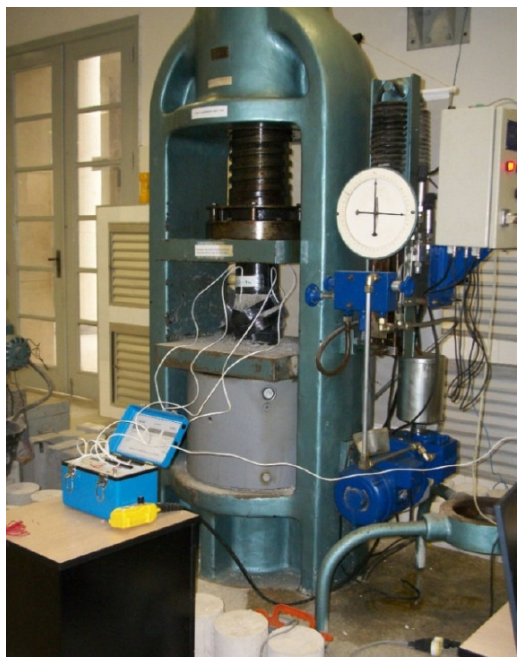

Figura 10: Instrumentação externa dos espécimes ensaiados

\section{RESULTADOS E ANÁLISE}

A Tabela 5 apresenta os valores médios de resistência, obtidos nos ensaios de compressão axial de três colunas curtas de concreto armado sem e com reforço externo colado $\left(\mathrm{f}_{\mathrm{co}}\right.$ e $\mathrm{f}_{\mathrm{cc}}$ ) por meio de compósito de resina e fibras de carbono.

Verifica-se na Tabela 5 que os ganhos de resistência média dos espécimes reforçados e com estribos a cada $60 \mathrm{~mm}$ em relação aos não reforçados e com estribos a cada $60 \mathrm{~mm}$ foram cerca de $119 \%$, para 1 camada de reforço, e 233\%, para 2 camadas de reforço, enquanto nos espécimes reforçados e com estribos a cada $30 \mathrm{~mm}$ estes ganhos passaram para $126 \%$ e $236 \%$. 
Também pode ser constatado na Tabela 5 que o ganho de resistência média dos espécimes reforçados com 2 camadas e com estribos a cada $60 \mathrm{~mm}$ em relação aos com 1 camada e com estribos a cada $60 \mathrm{~mm}$ foi aproximadamente $52 \%$, ao passo que este ganho para os espécimes reforçados com 2 camadas e com estribos a cada 30 mm em relação aos com 1 camada e com estribos a cada $30 \mathrm{~mm}$ foi perto de $49 \%$.

Tabela 5 - Valores médios de resistência das colunas ensaiadas

\begin{tabular}{|c|c|c|c|c|}
\hline \multirow{2}{*}{ Sem reforço } & \multicolumn{4}{|c|}{ Com reforço } \\
\hline & \multicolumn{2}{|c|}{1 camada de reforço } & \multicolumn{2}{|c|}{2 camadas de reforço } \\
\hline $\mathrm{f}_{\mathrm{co}}(\mathrm{MPa})$ & $\mathrm{f}_{\mathrm{cc}}(\mathrm{MPa})$ & $\mathrm{f}_{\mathrm{cc}} / \mathrm{f}_{\mathrm{co}}$ & $\mathrm{f}_{\mathrm{cc}}(\mathrm{MPa})$ & $\mathrm{f}_{\mathrm{cc}} / \mathrm{f}_{\mathrm{co}}$ \\
\hline \multicolumn{5}{|c|}{ Colunas curtas de concreto armado com estribos a cada $60 \mathrm{~mm}$} \\
\hline 28,8 & 63,2 & 2,19 & 96,0 & 3,33 \\
\hline \multicolumn{5}{|c|}{ Colunas curtas de concreto armado com estribos a cada $30 \mathrm{~mm}$} \\
\hline 31,9 & 72,0 & 2,26 & 107,2 & 3,36 \\
\hline
\end{tabular}

Os valores médios das deformações longitudinais e transversais, obtidos nos ensaios de compressão axial de três colunas curtas de concreto armado sem $\left(\varepsilon_{\mathrm{co}}\right.$ e $\left.\varepsilon_{\mathrm{cot}}\right)$ e com reforço externo colado $\left(\varepsilon_{\mathrm{cc}}\right.$ e $\left.\varepsilon_{\mathrm{cct}}\right)$ por meio de compósito de resina e fibras de carbono, podem ser vistos na Tabela 6. Ressalta-se que estes valores foram coletados dos ensaios imediatamente antes da ruptura do compósito.

Os aumentos de deformação última longitudinal dos espécimes reforçados e com estribos a cada 60 mm em relação aos não reforçados e com estribos a cada $60 \mathrm{~mm}$ foram cerca de 639\%, para 1 camada de reforço, e 693\%, para 2 camadas de reforço, enquanto estes aumentos para os espécimes reforçados e com estribos a cada 30 mm em relação aos não reforçados e com estribos a cada $30 \mathrm{~mm}$ foram $763 \%$ e $993 \%$, conforme se pode ver na Tabela 6.

Em se tratando dos espécimes reforçados e com estribos a cada $60 \mathrm{~mm}$, constata-se na Tabela 6 que os ganhos médios de deformação última transversal em relação aos espécimes não reforçados e com estribos a cada $60 \mathrm{~mm}$ foram aproximadamente $1175 \%$, para 1 camada de reforço, e $10383 \%$, para 2 camadas de reforço, ao passo que estes ganhos para espécimes reforçados e com estribos a cada $30 \mathrm{~mm}$ foram $914 \%$ e $779 \%$.

Tabela 6 - Valores médios de deformação última das colunas ensaiadas

\begin{tabular}{|c|c|c|c|c|c|c|c|c|c|}
\hline \multirow{2}{*}{\multicolumn{2}{|c|}{ Sem reforço }} & \multicolumn{8}{|c|}{ Com reforço } \\
\hline & & \multicolumn{4}{|c|}{ 1camada de reforço } & \multicolumn{4}{|c|}{2 camadas de reforço } \\
\hline$\varepsilon_{\mathrm{co}}(\%)$ & $\varepsilon_{\mathrm{cot}}(\%)$ & $\varepsilon_{\mathrm{cc}}(\%)$ & $\varepsilon_{\mathrm{cc}} / \varepsilon_{\mathrm{co}}$ & $\varepsilon_{\mathrm{cct}}(\%)$ & $\varepsilon_{\mathrm{cct}} / \varepsilon_{\mathrm{cot}}$ & $\varepsilon_{\mathrm{cc}}(\%)$ & $\varepsilon_{\mathrm{cc}} / \varepsilon_{\mathrm{co}}$ & $\varepsilon_{\mathrm{cct}}(\%)$ & $\varepsilon_{\mathrm{cct}} / \varepsilon_{\mathrm{cot}}$ \\
\hline \multicolumn{10}{|c|}{ Colunas curtas de concreto armado com estribos a cada $60 \mathrm{~mm}$} \\
\hline 2,8 & 0,8 & 20,7 & 7,39 & 10,2 & 12,75 & 22,2 & 7,93 & 9,1 & 11,38 \\
\hline \multicolumn{10}{|c|}{ Colunas curtas de concreto armado com estribos a cada $30 \mathrm{~mm}$} \\
\hline 2,7 & 1,4 & 23,3 & 8,63 & 14,2 & 10,14 & 29,5 & 10,93 & 12,3 & 8,79 \\
\hline
\end{tabular}

Nas Figura 11 e 12 podem ser visualizadas as curvas tensão longitudinal-deformação nas direções longitudinal e transversal dos espécimes sem e com reforço externo colado de compósito de resina e fibras de carbono.

Nessas curvas das Figuras 11 e 12 pode-se verificar que as resistências e, na maioria dos casos, as deformações últimas dos espécimes reforçados com duas camadas foram maiores que as dos reforçados com uma camada e estas maiores que as dos não reforçados.

As curvas tensão-deformação dos espécimes ensaiados não apresentaram ramo descendente, o que expressa o efeito favorável da seção transversal circular no caso de reforço com compósito.

Comparando as curvas entre os espécimes com estribos a cada $60 \mathrm{~mm}$ e os espécimes com estribos a cada $30 \mathrm{~mm}$, constata-se o efeito favorável quando se utilizam estribos de menor espaçamento. Ocorreram maiores valores de resistência e deformações. 

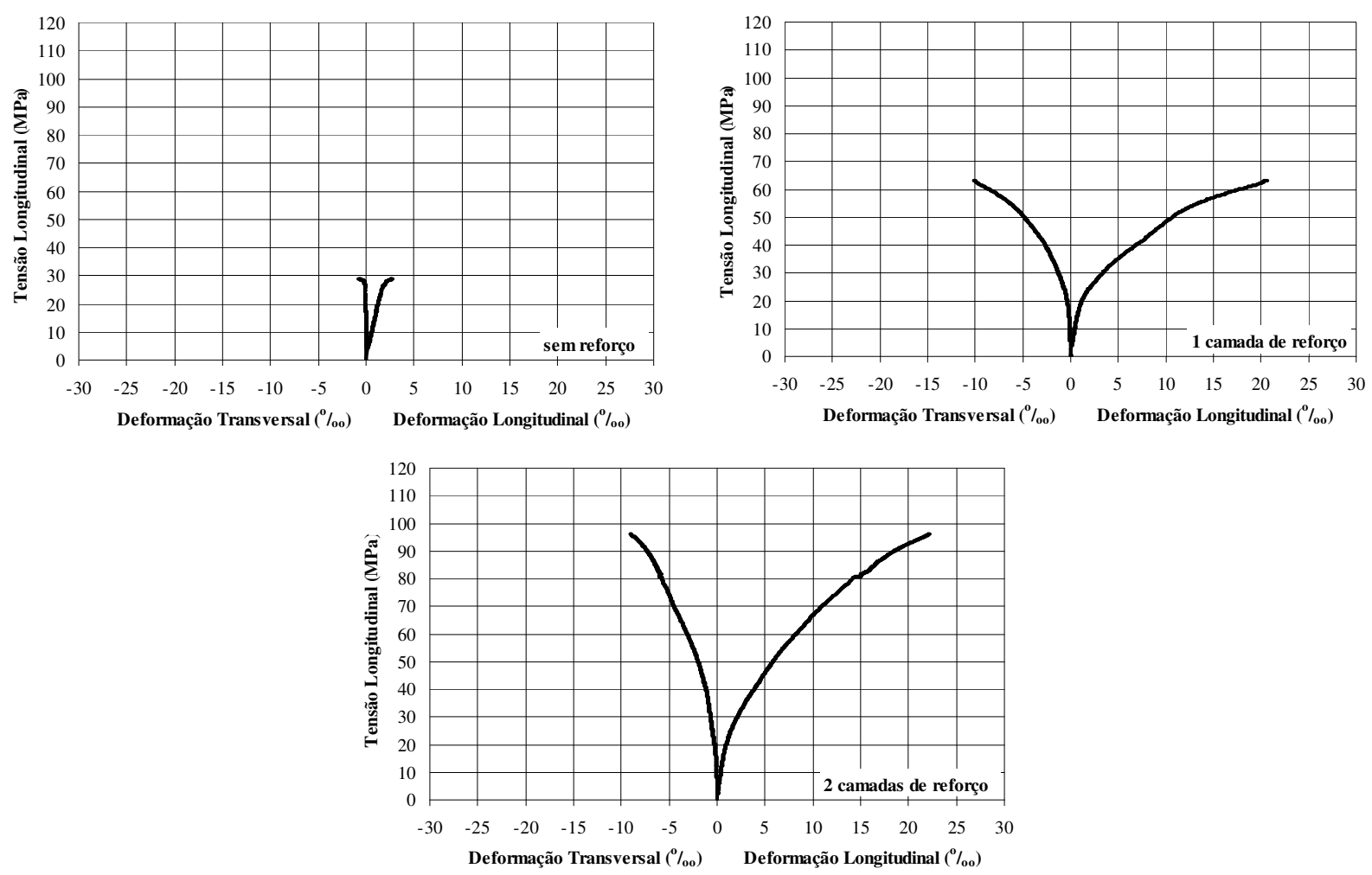

Figura 11: Cuvras tensão-deformação dos espécimes sem ou com reforço e com estribos a cada $60 \mathrm{~mm}$
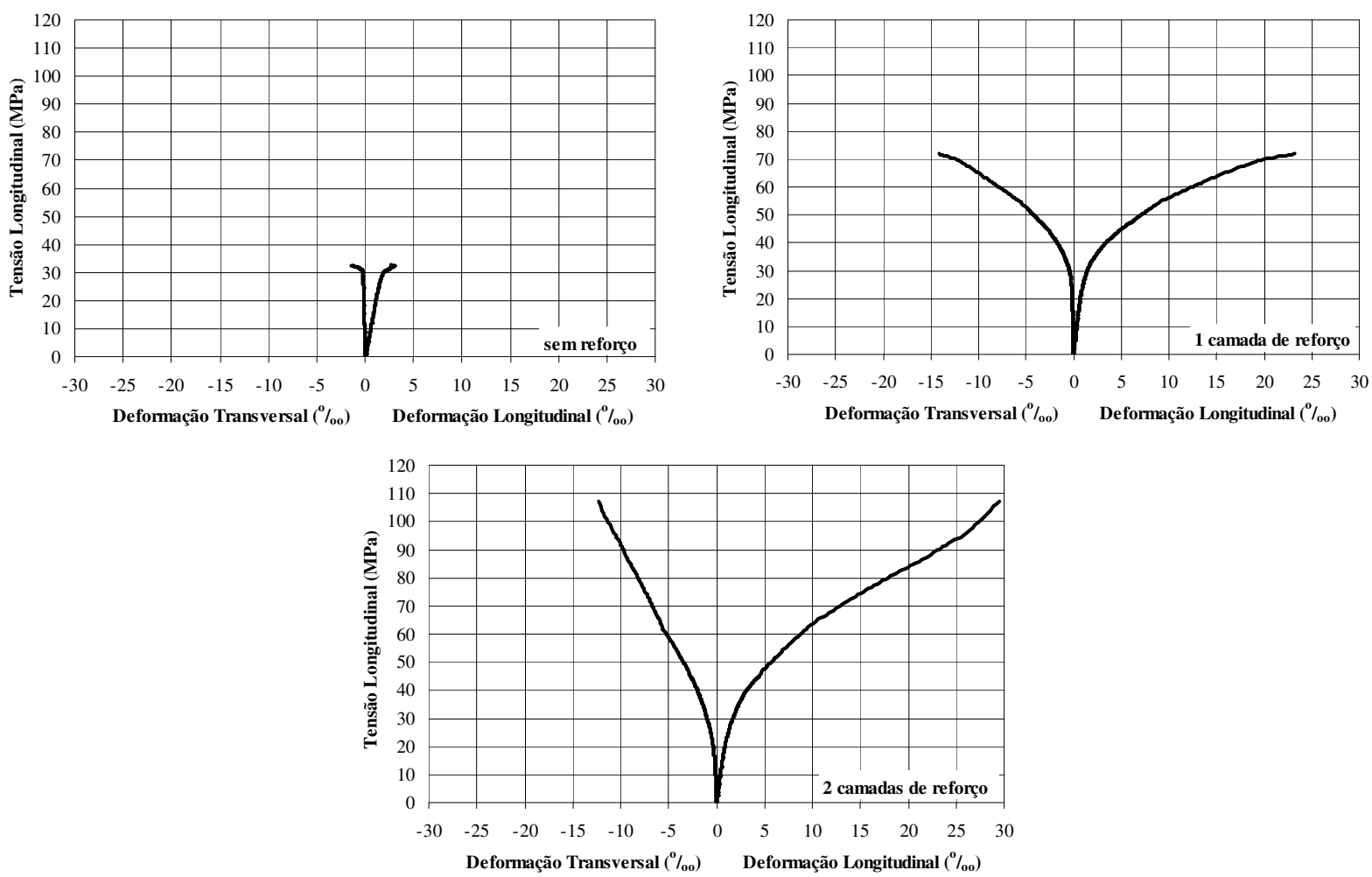

Figura 12: Cuvras tensão-deformação dos espécimes sem ou com reforço e com estribos a cada $30 \mathrm{~mm}$ 
Na Figura 13 podem ser visualizados os modos de ruptura dos espécimes sem ou com reforço de folha unidirecional de compósito de resina e fibras de carbono.

Estampidos foram ouvidos na iminência da ruptura frágil em todos os espécimes. O reforço rompeu na região à meia altura em todos os espécimes confinados com 1 camada de reforço, enquanto nos espécimes confinados com 2 camadas, em geral, a ruptura se deu na região entre a metade da altura e a face superior ou inferior.
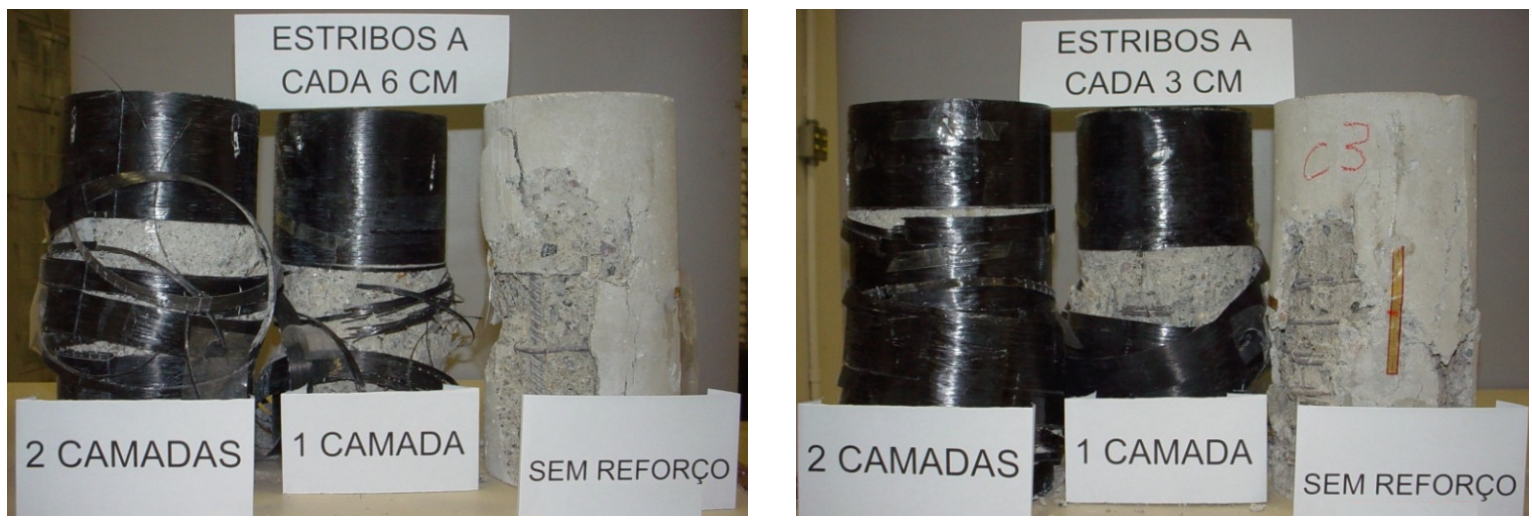

Figura 13: Colunas curtas de concreto armado sem e com reforço de CFRP após a ruptura

\section{CONSIDERAÇÕES FINAIS}

Dos resultados dos ensaios de 18 colunas curtas de concreto armado deste trabalho, das quais metade com espaçamento da armadura transversal de $30 \mathrm{~mm}$ e a metade restante com espaçamento de $60 \mathrm{~mm}$, sendo 12 reforçadas com uma ou duas camadas, e de alguns estudos sobre pilares de concreto coletados da literatura, pôde-se chegar às seguintes conclusões:

- as propriedades do concreto e do material compósito, a geometria, o raio de arredondamento das arestas e a taxa de armadura interna dos pilares são os parâmetros mais relevantes que influenciam o comportamento de pilares reforçados externamente com compósitos de resina e fibras de carbono;

- dentre as colunas curtas de concreto armado reforçadas com CFRP, as com estribos espaçados de $60 \mathrm{~mm}$ apresentaram ganhos de $119 \%$ ( 1 camada de reforço) e $233 \%$ ( 2 camadas de reforço), enquanto as com estribos espaçados de 30 mm, $126 \%$ ( 1 camada de reforço) e $236 \%$ ( 2 camadas de reforço);

- os ganhos médios de resistência das colunas curtas de concreto armado reforçadas com 2 camadas em relação aos reforçados com 1 camada foram 52\% (estribos a cada $60 \mathrm{~mm}$ ) e $49 \%$ (estribos a cada $30 \mathrm{~mm}$ );

- os valores de deformação última das colunas curtas de concreto armado reforçadas atingiram valores na ordem de $22 \%$ o (estribos espaçados a cada $60 \mathrm{~mm}$ ) e até $30 \%$ (estribos espaçados a cada $30 \mathrm{~mm}$ );

- os maiores ganhos médios de deformação última longitudinal foram de $993 \%$, para as colunas curtas de concreto armado com estribos espaçados de $30 \mathrm{~mm}$ e reforçadas com 2 camadas;

- as curvas tensão - deformação dos pilaretes de seção circular não apresentaram ramo descendente, o que expressa o efeito favorável deste tipo de seção transversal no caso de reforço com compósitos;

- quanto o menor espaçamento entre estribos em pilares de concreto armado sem ou com reforço de compósitos de resina e fibras de carbono, maior o aumento de resistência e de ductilidade, devido ao efeito favorável de confinamento do núcleo de concreto.

\section{REFERÊNCIAS}

ASSOCIAÇÃO BRASILEIRA DE NORMAS TÉCNICAS. NBR 5739: Concreto - Ensaio de Compressão de Corpos-de-Prova Cilíndricos, Rio de Janeiro, 2018.

AMERICAN SOCIETY OF TESTING MATERIALS. ASTM D 3039/D 3039 M: Standard Test Method for Tensile Proprieties of Polymer Matrix Composite Materials. Annual Book of ASTM Standards American Society of Testing Materials, West Conshohocken, 2000. 
BENZAID, R., MESBAH, H., CHIKH, N.E. FRP-confined Concrete Cylinders: Axial Compression Experiments and Strength Model. Journal of Reinforced Plastics and Composites, v. 29, n. 4, pp. 2469-2488, 2010.

CARnEIRO, L. A. V. Reforço de Vigas e Pilares de Concreto com Materiais Compósitos de Resina e Fibras. Tese de Doutorado, COPPE/UFRJ, Rio de Janeiro, 2004.

CHASTRE, C., SILVA, M. A. G. Monotonic axial behavior and modelling of RC circular columns confined with CFRP. Engineering Structures, v. 32, n. 8, pp. 2268-2277, 2010.

COLE, C., BELARBI, A. Confinement Characteristics of Rectangular FRP-Jacketed RC Columns, FibreReinforced Plastics for Reinforced Concrete Structures, In: Proceedings of the Fifth International Conference on FRPRCS, v. 1, pp. 823-832, Cambridge, UK, Jul., 2001.

DEMERS, M., NEALE, K., W. Confinement of Reinforced Concrete Columns with Fibre-Reinforced Composite Sheets - An Experimental Study. Canadian Journal of Civil Engineering, v. 26, n. 2, pp. 226-241, Apr., 1999.

DIOGO, T. C. D’ALMEIDA. Estudo Experimentaldo Reforço à Força Cortante de Corpos-de-prova de Concreto com Compósito de Fibras de Carbono. Dissertação de Mestrado da UFF, Niterói, 2010.

HOSOTAni, M., KAWASHIMA, K., HOSHIKUMA, J. A Study on Confinement Effect of Concrete Cylinders by Carbon Fiber Sheets. Non-Metallic (FRP) Reinforcement for Concrete Structures, In: Proceedings of the Third International Symposium, v. 1, pp. 209-216, Oct., 1997.

ILKI, A., PEKER, O., KARAMUK, E., DEMIR, C., KUMBASAR, N. FRP Retrofit of Low and Medium Strength Circular and Rectangular Reinforced Concrete Columns. Journal of Materials in Civil Engineering, v. 20, n. 2, pp. 169-188, Fev., 2008.

MAADDAWY, T., SAYED, M., ABDEL-MAGID, B. The effects of cross-sectional shape and loading condition on performance of reinforced concrete members confined with Carbon Fiber-Reinforced Polymers. Materials and Design, v. 31, n. 5, pp. 2330-2341., 2010.

MATTHYS, S. Structural Behaviour and Design of Concrete Members Strengthened with Externally Bonded FRP Reinforcement. D.Sc. Thesis, University of Ghent, Ghent, Belgium, 2000.

PAULA, R. F., SILVA, M. G., Sharp Edge Effects on FRP Confinement of RC Square Columns, In: Proceedings of Composites in Infrastructure ICCI, University of Arizona, San Francisco, California, USA, Jun., 2002.

PESSIKI, S., HARRIES, K. A., KESTNER, J. T., et al. Axial Behavior of Reinforced Concrete Columns Confined with FRP Jackets. Journal of Composites for Construction, v. 5, n. 4, pp. 237-245, Nov., 2001.

SILVA, M. A. G. Behavior of square and circular columns strengthened with aramidic or carbon fibers. Construction and Building Materials, v. 25, n. 8, pp. 3222-3228., 2011.

WANG, Y. C., RESTREPO, J. I. Investigation of Concentrically Loaded Reinforced Concrete Columns Confined with Glass Fiber-Reinforced Polymer Jackets. ACI Structural Journal, v. 98, n. 3, pp. 377-385, May-Jun., 2001. 